\title{
Long-term stability of the dead-zone in proto-planetary disks
}

\author{
Mario Flock ${ }^{1}$, Neal Turner ${ }^{1,2}$, Natalia Dzyurkevich ${ }^{1}$ and \\ Hubert Klahr ${ }^{1}$ \\ ${ }^{1}$ Max Planck Institute for Astronomy, Königstuhl 17, 69117 Heidelberg, Germany \\ email: flock@mpia-hd.mpg.de \\ ${ }^{2}$ Jet Propulsion Laboratory, California Institute of Technology, Pasadena, CA 91109, USA
}

\begin{abstract}
We present 3D global non-ideal MHD simulations with a self consistent dynamic evolution of ionization fraction of the gas as result of reduced chemical network. We include X-ray ionization from the star as well as cosmic ray ionization. Based on local gas density and temperature in our chemical network, we determine the magnetic resistivity, which is fed back in MHD simulations. Parameters for dust size and abundance are chosen to have accreting layers and a laminar "dead" mid-plane.
\end{abstract}

Keywords. accretion disks, MHD, turbulence, planetary systems: formation, instabilities

\section{Introduction}

Magneto-rotational instability (MRI) is the most prominent process to drive turbulence and angular momentum transport in protoplanetary disks (Balbus and Hawley 1998). To enable MRI to operate in disk, the ionization of gas has to be sufficiently high. Various studies showed that at a certain level of resistivity MRI will be suppressed, mostly due to the small sized dust grains which absorb free electrons (Turner 2010 and references therein). Most studies for MRI in stratified accretion disk rely on local box simulations as well as static ionization profiles of the disk. Until now, no global simulation with a dynamical resistivity profile was calculated. In our work we investigate how far MRI can change the resistivity profile by turbulent motion itself.

\section{Model}

Numerical setup follows the second order PLUTO configuration in Flock et al. (2010) with the HLLD Riemann solver. We use a locally isothermal global disk model with $\mathrm{H} / \mathrm{R}=0.05 ; \mathrm{r}: 3.6$ - 6.6 (128 grid cells); $\theta: \pm 5$ scale heights (160) and $\phi: 45^{\circ}$ (128). The hydrodynamical model follows Dzyurkevich et al. (2010). We use a weak vertical net field as seed for MRI. In the dead zone region this field is still visible after 500 local orbits (Fig. 1, right, vectors). For the chemical model (Turner et al. 2010), we have chosen dust size and abundance that the dead zone has an extent of \pm 2.5 scale heights at the beginning.

\section{Result}

The upper layers of the disk above 2 scale heights reach after 10 local orbits a quasi steady state. In Fig. 1, right, above 2.5 scale height there are a large scale turbulent structures in all 3 magnetic field components. The dark-violet line shows the Elsässer number $\left(\Lambda_{e l}\right)$ of unity around 2.5 scale heights. Radial average of this value stays at 2.5 

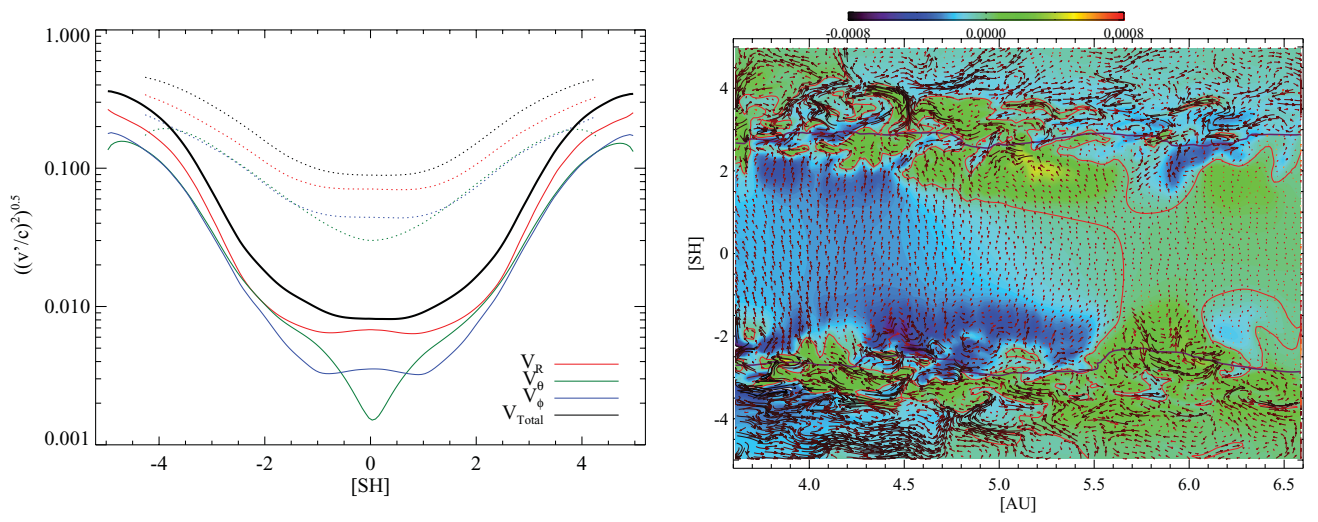

Figure 1. Left: Turbulent RMS velocities over height. Dotted lines present the turbulent RMS velocity for a full ionized and MRI turbulent disk. Solid line present the RMS velocity for our dead-zone run. Right: $r-\theta$ snapshot of the magnetic fields after 500 local orbits. The azimuthal component is plotted as contour color with red lines showing change of sign. Over-plotted are the respective radial and theta component as vector field. The dark-violet line present the Elsässer number of unity.

scale heights, even after 1000 local orbits. In addition we observe a quasi active dead zone region around 2 scale heights but with $\Lambda_{e l}<1$. Here, there is an accumulation of azimuthal magnetic field, antisymmetric for both hemispheres, as well as transport of material outward the dead zone. The midplane of dead zone actually shows a Mach number of 0.01 (Fig. 1, left). In addition we integrate the total mass flux through the vertical boundary at 5 scale height as well as the total radial mass flux at 4 and 6 AU. Even at 5 scale heights the vertical mass transport dominates the radial one by a factor of 3 (integrated until 1000 local orbits).

\section{Summary}

We do not see separation of the dead zone into rings in our dynamical model (i.e. no viscous instability of dead zone). The dead zone thickness is stable for over thousand of local orbits. In our simulation, evacuation timescale of dead zone is set by the vertical mass outflow. Total integrated mass which escapes the simulation at 5 scale heights is 3 times larger than total radial transport. This value is measured after 1000 orbits of evolution. Turbulent velocities at the midplane in dead zone are around $1 \%$ of local sound speed, which is one order of magnitude below the value for ideal MHD case.

\section{References}

Balbus, S. A. \& Hawley, J. F. 1998, Reviews of Modern Physics, 70, 1

Dzyurkevich, N., Flock, M., Turner, N. J., Klahr, H., \& Henning, T. 2010, A\&AA, 515, A70

Flock, M., Dzyurkevich, N., Klahr, H., \& Mignone, A. 2010, A\& A, 516, A26

Turner, N. J., Carballido, A., \& Sano, T. N. 2010, ApJ, 708, 188 